\title{
TRAJECTORIES ESCAPING TO INFINITY IN FINITE TIME
}

\author{
J.K. LANGLEY
}

\begin{abstract}
If the function $f$ is transcendental and meromorphic in the plane, and either $f$ has finitely many poles or its inverse function has a logarithmic singularity over $\infty$, then the equation $\dot{z}=f(z)$ has infinitely many trajectories tending to infinity in finite increasing time. MSC 2010: 30D30.
\end{abstract}

\section{InTRODUCTION}

This paper concerns the differential equation

$$
\dot{z}=\frac{d z}{d t}=f(z),
$$

in which the function $f$ is meromorphic in a plane domain $D$ : see, for example, $[3,4$, $5,7,8,9,12]$ for fundamental results concerning such flows. A trajectory for $(1)$ is a path $z(t)$ in $D$ with $z^{\prime}(t)=f(z(t)) \in \mathbb{C}$ for $t$ in some maximal interval $(\alpha, \beta) \subseteq \mathbb{R}$. The present paper is motivated by a result from [12] involving trajectories which tend to infinity in finite increasing time, that is, which satisfy $\beta \in \mathbb{R}$ and $\lim _{t \rightarrow \beta-} z(t)=\infty$. King and Needham [12, Theorem 5] showed that if $f$ has a pole at infinity of order at least 2 then such trajectories always exist for (1) (see Section 2). It seems reasonable to ask whether trajectories of this type must exist if $f$ is transcendental and meromorphic in the plane, and the following will be proved in Section 3.

Theorem 1.1. Let the function $f$ be transcendental and meromorphic in the plane, with finitely many poles. Then (1) has infinitely many pairwise disjoint trajectories each tending to infinity in finite increasing time.

The proof of Theorem 1.1 is based on the Wiman-Valiron theory [10], which shows that if $f$ is as in the hypotheses then there exist small neighbourhoods on which $f(z)$ behaves like a constant multiple of a large power of $z$. In the simple example $\dot{z}=$ $-\exp (-z)$, all trajectories satisfy $\exp (z(t))=\exp (z(0))-t$ and tend to infinity as $t$ increases, taking finite time to do so if and only if $\exp (z(0))$ is real and positive.

For meromorphic functions with infinitely many poles the situation is in general different. Let $g$ be a transcendental entire function of order of growth $\rho(g)<1 / 2$ and let $f=-i g / g^{\prime}$ in (1). Then each trajectory has $i \log g(z(t))=t+C$, and $\log |g(z(t))|=\operatorname{Im} C$, with $C$ constant. Because $\rho(g)<1 / 2$, the classical $\cos \pi \rho$ theorem [11] implies that $\min \{|g(z)|:|z|=r\}$ is unbounded as $r \rightarrow \infty$, and so all trajectories are bounded. In this example $\infty$ is an asymptotic value of $f$, since estimates for logarithmic derivatives from [6] imply that $g^{\prime}(z) / g(z)$ tends to 0 as $z$ tends to infinity outside a small exceptional set. However, Theorem 1.2 below will show that infinitely many disjoint trajectories tending to infinity in finite increasing time must exist if $f$ satisfies the stronger condition that the inverse function has a logarithmic singularity over $\infty$, which is defined as follows $[1,13]$. 
Let $f$ be any transcendental meromorphic function in the plane, let $M$ be real and positive, and let $U$ be a component of the set $\{z \in \mathbb{C}:|f(z)|>M\}$ with the following property: for some $z_{0} \in U$ with $w_{0}=f\left(z_{0}\right) \in \mathbb{C}$, a branch of the inverse function $z=f^{-1}(w)$ is defined near $w_{0}$, mapping $w_{0}$ to $z_{0}$, and admits unrestricted analytic continuation in the annulus $M<|w|<\infty$. If $v_{0}$ is chosen so that $e^{v_{0}}=w_{0}$ then a function $\phi(v)=f^{-1}\left(e^{v}\right)$ may be defined on a neighbourhood of $v_{0}$ and extends by the monodromy theorem to an analytic function on the half-plane $H$ given by $\operatorname{Re} v>\log M$. Then, by a well known classification theorem [13, p.287], there are two possibilities. First, if $\phi$ is not univalent on $H$ then $\phi$ has period $m 2 \pi i$ for some minimal positive integer $m$ and $U$ contains precisely one pole $z_{1}$ of $f$ of multiplicity $m$, while $z \rightarrow z_{1}$ as $f(z) \rightarrow \infty$ with $z \in U$. On the other hand, if $\phi$ is univalent on $H$, then $U$ contains no poles of $f$, but $U$ does contain a path tending to infinity on which $f(z)$ tends to infinity, and the inverse function $\log f$ of $\phi$ maps $U$ univalently onto $H$. In this second case the inverse function of $f$ is said to have a logarithmic singularity over $\infty$, and $U$ is called a neighbourhood of the singularity [1].

Theorem 1.2. Let the function $f$ be transcendental and meromorphic in the plane such that its inverse function $f^{-1}$ has a logarithmic singularity over $\infty$. Then for each neighbourhood $U$ of the singularity there exist infinitely many pairwise disjoint trajectories of the flow (1), on each of which $z(t)$ tends to infinity in finite increasing time with $z(t) \in U$.

Examples to which Theorem 1.2 applies include $f(z)=e^{-z^{2}} \tan z$ : here $\infty$ is an asymptotic value of $f$, but the finite critical and asymptotic values form a bounded set, so that the two singularities of $f^{-1}$ over $\infty$ are logarithmic. Theorem 1.2 follows from the next result.

Theorem 1.3. Let $f$ be a meromorphic function on a domain $\Omega \subseteq \mathbb{C}$ such that there exist a real number $M>0$, a domain $U \subseteq \Omega$ and an analytic function $F: U \rightarrow \mathbb{C}$ with the property that $f=e^{F}$ on $U$ and $F$ maps $U$ univalently onto the half-plane $H=\{w \in \mathbb{C}: \operatorname{Re} w>\log M\}$. Then (1) has infinitely many pairwise disjoint trajectories $z(t)$ on which $z(t)$ tends in finite increasing time from within $U$ to the extended boundary $\partial_{\infty} \Omega$ of $\Omega$.

Here the statement that a trajectory $z(t)$ tends in finite increasing time from within $U$ to the extended boundary of $\Omega$ means that there exists $T \in \mathbb{R}$ with the following property: to each compact set $K_{0} \subseteq \Omega$ corresponds $t_{0} \in(-\infty, T)$ with $z(t) \in U \backslash K_{0}$ for $t_{0}<t<T$. To deduce Theorem 1.2 from Theorem 1.3 it is only necessary to take $\Omega=\mathbb{C}$ and $U$ to be a neighbourhood of the logarithmic singularity of $f^{-1}$ over $\infty$, so that $|z(t)| \rightarrow+\infty$ as $t \rightarrow T-$.

\section{Preliminaries}

If the function $f$ is meromorphic and non-constant on a domain $D \subseteq \mathbb{C}$, and $w \in D$ with $f(w) \neq \infty$, then the trajectory of (1) through $w$ is the path $z(t)=\zeta_{w}(t) \in D$ with $z(0)=w$ and $z^{\prime}(t)=f(z(t)) \in \mathbb{C}$ for $t$ in some maximal interval $(\alpha, \beta) \subseteq \mathbb{R}$. If $f(w)=0$ then $\zeta_{w}(t)=w$ for all $t \in \mathbb{R}$. When $f(w) \neq 0$ the trajectory passes through no zeros of $f$, and is either simple (that is, $\zeta_{w}(t)$ is injective on $(\alpha, \beta)$ ) or periodic (in which case $(\alpha, \beta)=\mathbb{R})$.

Some standard facts concerning (1) near poles of $f$ will now be summarised: for details, see $[3,7,12]$. If $f(z) \sim c\left(z-z_{0}\right)^{-m}$ as $z \rightarrow z_{0}$, for some $c \neq 0$ and $m \geq 0$, 
then a conformal mapping $w=\phi(z)$ is defined near $z_{0}$ by $\phi(z)^{m+1}=\int_{z_{0}}^{z} 1 / f(u) d u$, which gives $(m+1) w^{m} \dot{w}=1$ and $w^{m+1}(t)=w^{m+1}(0)+t$. The equation for $w$ has $m+1$ pairwise disjoint trajectories tending to 0 in increasing time, determined by choosing $w^{m+1}(0) \in(-\infty, 0) \subseteq \mathbb{R}$. Thus (1) has precisely $m+1$ trajectories tending to $z_{0}$ in increasing time (each taking finite time to do so).

If $D$ contains an annulus $R<|z|<\infty$ and $f$ has a pole of order $n \geq 2$ at infinity, then setting $w=1 / z$ gives $\dot{w}=g(w)=-f(z) / z^{2}$, so that $g$ has a pole of order $n-2$ at $w=0$ and (1) has $n-1$ trajectories tending to infinity in finite increasing time: this proves the result of King and Needham [12] referred to in the introduction.

Theorem 1.1 requires the following lemma: a proof is included for completeness.

Lemma 2.1. Let the function $f$ be meromorphic and non-constant on $\mathbb{C}$. Let $z(t)$ be a trajectory of (1), with maximal interval of definition $\left(a_{0}, b_{0}\right) \subseteq \mathbb{R}$, and assume

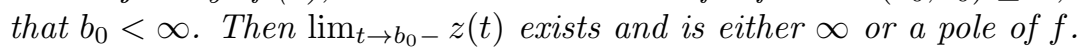

Proof. Following [8], a point $z_{0} \in \mathbb{C} \cup\{\infty\}$ is called a limit point of $z(t)$ as $t \rightarrow b_{0}-$ if there exist $s_{n} \in\left(a_{0}, b_{0}\right)$ with $s_{n} \rightarrow b_{0}-$ and $z\left(s_{n}\right) \rightarrow z_{0}$ as $n \rightarrow \infty$. Suppose that $z_{0} \in \mathbb{C}$ with $f\left(z_{0}\right) \neq 0, \infty$ is such a limit point. Writing $u(t)=\phi(z(t))$, where $\phi(z)=\int_{z_{0}}^{z} 1 / f(s) d s$, transforms (1) near $z_{0}$ to $\dot{u}=1$. Let $\rho$ be small and positive and let $U=\phi^{-1}(B(0,2 \rho))$ and $V=\phi^{-1}(B(0, \rho))$, with $B(a, r)$ the open disc of centre $a$ and radius $r$. Then any trajectory of (1) which meets $V$ must subsequently travel from the boundary of $V$ to that of $U$, taking time at least $\rho$ to do so. Since $b_{0}$ is finite this implies that $z(t) \rightarrow z_{0}$ as $t \rightarrow b_{0}-$, and that the trajectory extends beyond time $t=b_{0}$, contrary to assumption. Thus any finite limit point $z_{0}$ of $z(t)$ as $t \rightarrow b_{0}-$ has $f\left(z_{0}\right) \in\{0, \infty\}$.

It follows that if $z_{0} \in \mathbb{C} \cup\{\infty\}$ is a limit point of $z(t)$ as $t \rightarrow b_{0}-$, then

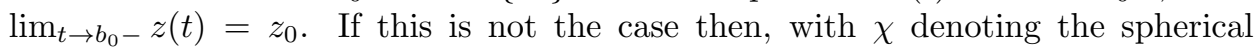
metric on the extended complex plane, there exists a small positive $\sigma$ such that $f(z) \neq 0, \infty$ on $X=\left\{z \in \mathbb{C}: \chi\left(z, z_{0}\right)=\sigma\right\}$ and $z(t)$ meets $X$ infinitely often as $t \rightarrow b_{0}-$. But this gives $z_{0}^{\prime} \in X$ such that $z_{0}^{\prime}$ is a limit point of $z(t)$ as $t \rightarrow b_{0}-$, and hence a contradiction.

It remains only to note that if $z_{0}$ is a zero of $f$ then it takes infinite time for any trajectory of (1) to tend to $z_{0}$. To see this, take $C>0$ and $m \in \mathbb{N}$ such that $|f(z)| \leq C\left|z-z_{0}\right|^{m}$ as $z \rightarrow z_{0}$. Let $n$ be large and take any trajectory $z(t)$ such that $\left|z\left(t_{n}\right)-z_{0}\right|=2^{-n}$ and $\left|z\left(t_{n+1}\right)-z_{0}\right|=2^{-n-1}$ and $2^{-n-1} \leq\left|z(t)-z_{0}\right| \leq 2^{-n}$ for $t_{n} \leq t \leq t_{n+1}$. This yields

$$
2^{-n-1} \leq\left|z\left(t_{n+1}\right)-z\left(t_{n}\right)\right|=\left|\int_{t_{n}}^{t_{n+1}} f(z(t)) d t\right| \leq\left(t_{n+1}-t_{n}\right) C 2^{-n m}
$$

and so $t_{n+1}-t_{n} \geq C^{-1} 2^{(m-1) n-1} \geq 1 / 2 C$.

The remainder of this section will be occupied with the proof of the following.

Proposition 2.1. Let the function $f$ be transcendental and meromorphic in the plane, and assume the existence of an unbounded set $F_{1} \subseteq[1, \infty)$ and a function $N(r): F_{1} \rightarrow[1, \infty)$ with

$$
\lim _{r \rightarrow \infty, r \in F_{1}} N(r)=\infty
$$


such that for each $r \in F_{1}$ there exists $z_{r}$ with $\left|z_{r}\right|=r$ and $f\left(z_{r}\right) \neq 0$ and

$$
f(z)=(1+o(1))\left(\frac{z}{z_{r}}\right)^{N(r)} f\left(z_{r}\right) \text { on } D\left(z_{r}, 8\right),
$$

as $r \rightarrow \infty$ in $F_{1}$, where

$$
D\left(z_{r}, L\right)=\left\{z_{r} e^{\tau}: \max \{|\operatorname{Re} \tau|,|\operatorname{Im} \tau|\} \leq L N(r)^{-5 / 8}\right\} .
$$

Then for all sufficiently large $r \in F_{1}$ there exist $Q \geq N(r)^{1 / 4}$ points $Y_{1}, \ldots, Y_{Q}$ in $D\left(z_{r}, 1\right)$, each with the property that the trajectory $\gamma_{j}=\zeta_{Y_{j}}$ with $\zeta_{Y_{j}}(0)=Y_{j}$ of (1) has maximal interval of definition $\left(\alpha_{Y_{j}}, \beta_{Y_{j}}\right)$, where

$$
\beta_{Y_{j}} \leq P_{r}=\frac{2 r}{\left|f\left(z_{r}\right)\right|(N(r)-1) \exp \left(N(r)^{1 / 4}\right)} .
$$

These trajectories $\gamma_{j}$ are pairwise disjoint.

To prove Proposition 2.1, let $r \in F_{1}$ be large, let $N=N(r)$ and define $w_{r}$ by $w_{r}=z_{r} \exp \left(4 N^{-5 / 8}\right)$. Then (2), (3) and Cauchy's estimate for derivatives yield

$$
\begin{aligned}
& A(z)=\frac{1}{f(z)}=\left(\frac{z}{w_{r}}\right)^{-N} A\left(w_{r}\right)(1+\mu(z)), \\
& \mu(z)=o(1), \quad \mu^{\prime}(z)=o\left(\frac{N^{5 / 8}}{r}\right),
\end{aligned}
$$

uniformly for $z$ in $D\left(z_{r}, 4\right)$. Again for $z$ in $D\left(z_{r}, 4\right)$, set

$$
\begin{aligned}
Z & =F(z)=\frac{w_{r} A\left(w_{r}\right)}{1-N}+\int_{w_{r}}^{z} A(t) d t \\
& =\frac{w_{r} A\left(w_{r}\right)}{1-N}+\int_{w_{r}}^{z}\left(\frac{t}{w_{r}}\right)^{-N} A\left(w_{r}\right)(1+\mu(t)) d t,
\end{aligned}
$$

and let $\sigma_{z}$ be the path from $w_{r}$ to $z$ which consists of the radial segment from $w_{r}$ to $\widehat{z}=w_{r}\left|z / w_{r}\right|$ followed by the shorter circular arc from $\widehat{z}$ to $z$. Then $\sigma_{z}$ has length $O\left(r N^{-5 / 8}\right)$ and $\left|w_{r}\right| \geq|t| \geq|z|$ on $\sigma_{z}$, so (6) and integration by parts along $\sigma_{z}$ yield

$$
\int_{w_{r}}^{z} t^{-N} \mu(t) d t=o\left(\frac{|z|^{1-N}}{N-1}\right)-\int_{w_{r}}^{z} o\left(\frac{N^{5 / 8}}{r}\right) \frac{t^{1-N}}{1-N} d t=o\left(\frac{|z|^{1-N}}{N-1}\right) .
$$

Hence $Z$ satisfies, still for $z \in D\left(z_{r}, 4\right)$, using (3) and (7),

$$
\begin{aligned}
Z & =F(z) \sim \frac{z^{1-N} A\left(w_{r}\right)}{w_{r}^{-N}(1-N)} \sim \frac{z^{1-N} A\left(z_{r}\right)}{z_{r}^{-N}(1-N)}, \\
|Z| & \sim\left|\frac{z}{r}\right|^{1-N} T_{r}, \quad T_{r}=\frac{r\left|A\left(z_{r}\right)\right|}{N-1},
\end{aligned}
$$

and

$$
\log Z=(1-N) \log \frac{z}{z_{r}}+\log \frac{z_{r} A\left(z_{r}\right)}{1-N}+o(1)
$$

where $\log \left(z / z_{r}\right)$ is chosen so as to vanish at $z_{r}$, and $\log \left(z_{r} A\left(z_{r}\right) /(1-N)\right)$ is the principal value. 
Lemma 2.2. Any sub-trajectory $\Lambda \subseteq D\left(z_{r}, 4\right)$ of the flow (1) is a level curve on which $\operatorname{Im} F(z)$ is constant and $\operatorname{Re} F(z)$ increases in increasing time. If $\Lambda$ joins $w_{0}$ to $w_{1}$ then the time taken for the flow (1) to traverse $\Lambda$ is

$$
\int_{w_{0}}^{w_{1}} \frac{d t}{d z} d z=\int_{w_{0}}^{w_{1}} \frac{1}{f(z)} d z=F\left(w_{1}\right)-F\left(w_{0}\right) .
$$

Let $Q=Q_{r}$ be the largest positive integer not exceeding $2 N^{1 / 4}$. Then provided $r \in F_{1}$ is large enough there exists a domain $\Omega_{r}$, the closure of which lies in $D\left(z_{r}, 1\right)$, such that $Y=\log Z$ maps $\Omega_{r}$ univalently onto the rectangle

$$
\begin{aligned}
G_{r} & =\left\{Y \in \mathbb{C}: \log S_{r}<\operatorname{Re} Y<\log T_{r}, \quad 0<\operatorname{Im} Y<4 Q \pi\right\}, \\
S_{r} & =T_{r} \exp \left(-N^{1 / 4}\right)=\frac{P_{r}}{2},
\end{aligned}
$$

and $S_{r}=o\left(T_{r}\right)$ as $r \rightarrow \infty$ with $r \in F_{1}$. The boundary of $\Omega_{r}$ contains a simple arc $L_{r}$ such that, as $z$ describes the arc $L_{r}$ once, the image $w=Z=F(z)$ describes $2 Q$ times the circle $|w|=S_{r}$, starting from $w=S_{r}$. Moreover, $\Omega_{r}$ contains $2 Q$ pairwise disjoint simply connected domains $V_{r}^{1}, \ldots, V_{r}^{2 Q}$, each mapped univalently by $F$ onto $\left\{w \in \mathbb{C}: S_{r}<|w|<T_{r}, 0<\arg w<2 \pi\right\}$. These domains have the following additional properties.

Let $V_{r}$ be any one of the $V_{r}^{j}$. Then $\partial V_{r}$ consists of the following: two simple arcs $I_{r} \subseteq L_{r}$ and $J_{r}$ mapped by $F$ onto the circles $|w|=S_{r}$ and $|w|=T_{r}$ respectively; two sub-trajectories of (1) mapped by $F$ onto the interval $\left[S_{r}, T_{r}\right]$.

Proof. The first two assertions hold because writing $Z=F(z)$ gives $\dot{Z}=1$. The existence of $\Omega_{r}, L_{r}$ and the $V_{r}^{j}$ follows from (8) and (9), which imply that $\log Z$ is a univalent function of $\log z$ on $D\left(z_{r}, 7 / 2\right)$. In particular, $L_{r}$ is the pre-image under $\log Z$ of $\left\{\log S_{r}+i \sigma: 0 \leq \sigma \leq 4 Q \pi\right\}$. Finally, (2), (5), (6), (8) and (10) give $P_{r}=2 S_{r}=o\left(T_{r}\right)$.

Assume henceforth that $r \in F_{1}$ is so large that Lemma 2.2 gives $P_{r}=2 S_{r}<T_{r}-S_{r}$. Choose some $V_{r}=V_{r}^{j}$ and let $W_{r}$ be the closure of $V_{r}$. The next lemma describes the behaviour of the trajectory $\zeta_{w}(t)$ of $(1)$ through $\zeta_{w}(0)=w \in I_{r}$.

Lemma 2.3. Suppose that $w \in I_{r}$ and $\operatorname{Re} F(w) \geq 0$. Then there exists $t_{w} \geq T_{r}-S_{r}$ such that $\zeta_{w}(t) \in W_{r} \backslash\left(J_{r} \cup I_{r}\right)$ for $0<t<t_{w}$, while $\zeta_{w}\left(t_{w}\right) \in J_{r}$. If $\operatorname{Re} F(w)>0$ and $t<0$ and $|t|$ is small, then $\left|F\left(\zeta_{w}(t)\right)\right|<S_{r}$.

Similarly, if $w \in I_{r}$ and $\operatorname{Re} F(w) \leq 0$, there exists $t_{w} \leq S_{r}-T_{r}$ such that $\zeta_{w}(t) \in W_{r} \backslash\left(J_{r} \cup I_{r}\right)$ for $t_{w}<t<0$, while $\zeta_{w}\left(t_{w}\right) \in J_{r}$. If $\operatorname{Re} F(w)<0$ and $t>0$ is small, then $\left|F\left(\zeta_{w}(t)\right)\right|<S_{r}$. If $w \in I_{r}$ and $\operatorname{Re} F(w)=0$, then $\zeta_{w}(t)$ travels from $w$ to $J_{r}$ via $W_{r}$ in both increasing and decreasing time.

Proof. Let $w \in I_{r}$ and $\operatorname{Re} F(w) \geq 0$. Then $|F(w)|=S_{r}$ and, for small positive $t$, both of $\operatorname{Re} F\left(\zeta_{w}(t)\right)$ and $\left|F\left(\zeta_{w}(t)\right)\right|$ are increasing, while $\operatorname{Im} F\left(\zeta_{w}(t)\right)$ is constant; thus $\zeta_{w}(t)$ remains within $W_{r}$ until it exits via $J_{r}$. The time taken to pass from $w$ to the first encounter with $J_{r}$, at $W$ say, is $F(W)-F(w)=|F(W)-F(w)| \geq T_{r}-S_{r}$. The remaining assertions are proved similarly.

Definition 2.1. For $u \in \mathbb{C}$ let $u^{*}$ denote the reflection of $u$ across the imaginary axis. A point $w \in I_{r}$ will be called recurrent if $\operatorname{Re} F(w)<0$ and there exists $t^{\prime}>0$ such that: (i) $\zeta_{w}(t)$ is defined for $0 \leq t \leq t^{\prime}$ and $w^{\prime}=\zeta_{w}\left(t^{\prime}\right) \in I_{r}$; (ii) $F\left(w^{\prime}\right)=F(w)^{*} ;$ (iii) $\zeta_{w}(t) \notin L_{r}$ for $0<t<t^{\prime}$; (iv) the Jordan curve $\Gamma_{w}$, formed 
from the arc of $I_{r}$ joining $w$ to $w^{\prime}$ and the sub-trajectory $\zeta_{w}(t), 0 \leq t \leq t^{\prime}$, encloses no zeros and no poles of $f$.

Since $F$ is univalent on $V_{r}$, and maps $I_{r}$ onto the circle $|w|=S_{r}$, with the endpoints of $I_{r}$ mapped to $S_{r}$, it follows that for $w, w^{\prime} \in I_{r}$ the equation $F\left(w^{\prime}\right)=F(w)^{*}$ determines $w^{\prime}$ uniquely from $w$, except when $F(w)=-S_{r}$. The next lemma follows at once from Lemma 2.2 and Cauchy's theorem applied to $1 / f$ and $\Gamma_{w}$.

Lemma 2.4. If $w \in I_{r}$ is recurrent then $t^{\prime} \leq\left|F\left(w^{\prime}\right)-F(w)\right| \leq 2 S_{r}=P_{r}$.

Lemma 2.5. If $w \in I_{r}$ with $\operatorname{Re} F(w)<0$ and $F(w)$ close to $\pm i S_{r}$, then $w$ is recurrent.

Proof. By the construction of $V_{r}$, the point $w$ lies in a small neighbourhood $\widehat{U}$ of some $\widehat{w} \in I_{r}$ with $F(\widehat{w})= \pm i S_{r}$ and $F$ univalent on $\widehat{U}$. Hence, as $\zeta$ describes $\zeta_{w}$ in increasing time, the image $F(\zeta)$ traverses the horizontal chord from $F(w)$ to $F(w)^{*}$ and $\zeta$ remains within $\widehat{U}$; thus $\zeta$ returns to meet $L_{r}$ at $w^{\prime} \in I_{r}$ with $F\left(w^{\prime}\right)=F(w)^{*}$. Therefore $w$ is recurrent.

Lemma 2.5 implies that the set of recurrent $w \in I_{r}$ is non-empty, and it follows from the next lemma that, for all but at most two $V_{r}^{j}$, the absence of $Y_{j}$ as in the conclusion of Proposition 2.1 forces all $v \in I_{r}$ with $\operatorname{Re} F(v)<0$ to be recurrent.

Lemma 2.6. Let $V_{r}=V_{r}^{j}$ be such that neither end-point of the arc $L_{r}$ lies in $W_{r}$, and assume that no $y \in I_{r}$ is such that $\operatorname{Re} F(y)<0$ and $\zeta_{y}$ has maximal interval of definition $\left(\alpha_{y}, \beta_{y}\right)$ with $\beta_{y} \leq P_{r}=2 S_{r}$. Then the following statements hold.

(a) Let $w \in I_{r}$ be such that $\operatorname{Re} F(w)<0$ and there exists a sequence $\left(w_{n}\right)$ in $I_{r}$ for which $w_{n} \rightarrow w$ as $n \rightarrow \infty$ and each $w_{n}$ is recurrent. Then $w$ is recurrent and $w_{n}^{\prime} \rightarrow w^{\prime}$ as $n \rightarrow \infty$.

(b) All $v \in I_{r}$ with $\operatorname{Re} F(v)<0$ are recurrent.

Proof. Let $w$ be as in (a), and observe that $F(w)^{*} \neq F(w)$, since $\operatorname{Re} F(w)<0$, and that $\zeta_{w}(t) \notin L_{r}$ for small positive $t$, by Lemma 2.3. By assumption, $\zeta_{w}$ has maximal interval of definition $\left(\alpha_{w}, \beta_{w}\right)$ with $\beta_{w}>2 S_{r}$.

Suppose first that there exists $\delta$ such that (11) $\left|\zeta_{w}(t)-u\right| \geq 2 \delta>0$ for all $u \in I_{r}$ with $F(u)=F(w)^{*}$ and all $t \in\left[0,2 S_{r}\right]$.

Note here that there exist at most two $u \in I_{r}$ with $F(u)=F(w)^{*}$. Since $w_{n} \rightarrow w$ and $w_{n}$ is recurrent it follows that $F\left(w_{n}^{\prime}\right)=F\left(w_{n}\right)^{*} \rightarrow F(w)^{*}$, and so $w_{n}^{\prime}$, for each large $n$, is close to some $u \in I_{r}$ with $F(u)=F(w)^{*}$. But (11) and continuous dependence on starting conditions now imply that if $n$ is large then

$$
\left|\zeta_{w_{n}}(t)-w_{n}^{\prime}\right| \geq \delta \text { for } 0 \leq t \leq 2 S_{r}
$$

This contradicts the fact that Definition 2.1 and Lemma 2.4 give $w_{n}^{\prime}=\zeta_{w_{n}}\left(t_{n}^{\prime}\right)$, where $0<t_{n}^{\prime} \leq 2 S_{r}$. Hence (11) cannot hold, and there exists a minimal $s$ with

$$
0<s \leq 2 S_{r}, \quad W=\zeta_{w}(s) \in L_{r},
$$

because if this is not the case then (11) evidently holds for some choice of $\delta$.

Suppose that $\operatorname{Re} F(W) \leq 0$, and take $k$ (possibly with $k \neq j$ ) such that $W \in \partial V_{r}^{k}$. Since $s \leq 2 S_{r}<T_{r}-S_{r}$, applying Lemma 2.3 to this $V_{r}^{k}$ shows that $w=\zeta_{w}(0)=$ $\zeta_{W}(-s) \notin L_{r}$, a contradiction.

Thus $W=\zeta_{w}(s) \in L_{r}$ and $\operatorname{Re} F(W)$ is positive. Suppose that $W \notin I_{r}$ or $F(W) \neq F(w)^{*}$, and take any $u \in I_{r}$ with $F(u)=F(w)^{*}$. Then Lemma 2.3 
(applied possibly to a different $V_{r}^{k}$ ) and the minimality of $s$ in (12) give $\zeta_{w}(t) \neq u$ for $0 \leq t \leq x=s+T_{r}-S_{r}$. Since $x>2 S_{r}$ there must exist $\delta$ such that (11) holds, which is impossible. This proves that $W=\zeta_{w}(s) \in I_{r}$ and $F(W)=F(w)^{*}$, so that $w$ satisfies conditions (i) to (iii) of Definition 2.1, with $t^{\prime}=s$ and $w^{\prime}=W$.

Now take any sequence $\left(x_{n}\right)$ in $I_{r}$ with $x_{n} \rightarrow w$ as $n \rightarrow \infty$. The trajectory $\zeta_{w}$ meets $L_{r}$ non-tangentially at $w$ and $W$, because $|F(z)|=S_{r}$ on $L_{r}$ and $Z=F(z)$ gives $\dot{Z}=1$ locally. Take a small positive $\rho$ and let $n \in \mathbb{N}$ be large. Then $\zeta_{w}(t)$ does not meet $L_{r}$ for $\rho \leq t \leq s-\rho$, by the minimality of $s$ in (12), and nor does $\zeta_{x_{n}}(t)$, by continuous dependence on initial conditions. Moreover, for $0 \leq t \leq \rho$, the trajectory $\zeta_{x_{n}}(t)$ follows a level curve on which $\operatorname{Im} F$ is constant, from $x_{n}$ to $\zeta_{x_{n}}(\rho)$, in which $F\left(\zeta_{x_{n}}(\rho)\right)=F\left(x_{n}\right)+\rho$. Furthermore, $\zeta_{x_{n}}(s-\rho)$ is close to $\zeta_{w}(s-\rho)$, which satisfies $F\left(\zeta_{w}(s-\rho)\right)=F(W)-\rho$. Thus for $t-s+\rho$ small and positive, $\zeta_{x_{n}}(t)$ again follows a level curve of $\operatorname{Im} F$, meeting $L_{r}$ non-tangentially at some point $x_{n}^{\prime \prime}$ near to $W$, using the fact that $W$ is not an end-point of $L_{r}$. Therefore $\zeta_{x_{n}}$ follows close to $\zeta_{w}$ and returns for the first time to $L_{r}$ at $x_{n}^{\prime \prime}$.

Applying this argument with $x_{n}=w_{n}$ shows that $w_{n}^{\prime}=x_{n}^{\prime \prime} \rightarrow W=w^{\prime}$, and that if $\Gamma_{w}$ is as in Definition 2.1 then, for large $n$, each point of $\Gamma_{w_{n}}$ lies close to $\Gamma_{w}$. Thus $w$ also satisfies condition (iv), and is recurrent. This proves part (a).

To prove part (b), observe that $I_{r}$ has relatively open subsets $U^{+}, U^{-}$, mapped by $\arg F(z)$ onto $(\pi / 2, \pi)$ and $(-\pi,-\pi / 2)$ respectively. Let $U_{0}$ be one of $U^{+}, U^{-}$; then $\widehat{U}_{0}=\left\{w \in U_{0}: w\right.$ is recurrent $\} \neq \emptyset$, by Lemma 2.5. Suppose that $U_{0} \neq \widehat{U}_{0}$. Then there exists some $v \in U_{0}$ which is a boundary point of $\widehat{U}_{0}$ relative to $U_{0}$; thus $v \in I_{r}$ with $\operatorname{Re} F(v)<0$ and $F(v) \neq-S_{r}$ and there are sequences $w_{n} \rightarrow v, v_{n} \rightarrow v$, with $w_{n}, v_{n} \in I_{r}$, such that each $w_{n}$ is recurrent, while each $v_{n}$ is not. By (a), $v$ is recurrent. For large $n$ the argument in the proof of (a), with $x_{n}=v_{n}, w=v$ and $W=v^{\prime}$, shows that $\zeta_{v_{n}}$ returns to meet $L_{r}$ for the first time after leaving $v_{n}$, at some $u_{n}=x_{n}^{\prime \prime} \in I_{r}$ close to $v^{\prime}$, without looping around any zeros or poles of $f$. But then Cauchy's theorem gives $\operatorname{Im}\left(F\left(u_{n}\right)-F\left(v_{n}\right)\right)=0$ and $F\left(u_{n}\right)=F\left(v_{n}\right)^{*}$, so that $v_{n}$ is recurrent, a contradiction. Hence all $v \in I_{r}$ with $\operatorname{Re} F(v)<0$ and $F(v) \neq-S_{r}$ are recurrent, and the same holds when $F(v)=-S_{r}$, by part (a).

Lemma 2.7. Let $V_{r}=V_{r}^{j}$ be such that neither end-point of the arc $L_{r}$ lies in $W_{r}$. Then there exists $y \in I_{r}$ such that $\operatorname{Re} F(y)<0$ and $\zeta_{y}$ has maximal interval of definition $\left(\alpha_{y}, \beta_{y}\right)$ with $\beta_{y} \leq P_{r}=2 S_{r}$.

Proof. Assume that this is not the case, and consider the unique $w \in I_{r}$ with $F(w)=-S_{r}$. Then Lemma 2.6 shows that $w$ is recurrent, and so $w^{\prime}$ is one of the two points $u_{1}, u_{2}$ on $I_{r}$ with $F\left(u_{j}\right)=S_{r}$; label these so that $w^{\prime}=u_{1}$. Choose a sequence $v_{n} \in I_{r}$ with $v_{n} \rightarrow u_{2}, v_{n} \neq u_{2}$, and for large $n$ choose the unique $w_{n} \in I_{r}$ with $F\left(w_{n}\right)=F\left(v_{n}\right)^{*} \rightarrow F\left(u_{2}\right)^{*}=-S_{r}$. Thus $w_{n} \rightarrow w, w_{n} \neq w$, and $w_{n}$ is recurrent for large $n$, by Lemma 2.6. But this gives $w_{n}^{\prime}=v_{n} \rightarrow u_{2} \neq w^{\prime}$, contradicting Lemma 2.6.

It follows from Lemma 2.7 that, for large $r \in F_{1}$, at least $2 Q-2 \geq Q \geq N^{1 / 4}$ of the domains $V_{r}^{1}, \ldots, V_{r}^{2 Q}$ give rise to pairwise distinct $Y_{j} \in \partial V_{r}^{j} \cap L_{r}$ such that $\operatorname{Re} F\left(Y_{j}\right)<0$ and $\zeta_{Y_{j}}$ has maximal interval of definition $\left(\alpha_{Y_{j}}, \beta_{Y_{j}}\right)$, in which $\beta_{Y_{j}}$ satisfies (5). Suppose that these trajectories are not pairwise disjoint. Then there exist distinct $j$ and $k$ such that $Y_{j}=\zeta_{Y_{k}}(S)$ and $Y_{k}=\zeta_{Y_{j}}(-S) \in L_{r}$ for some $S$ with $0<S<P_{r}$. But Lemma 2.3 shows that $\zeta_{Y_{j}}(t) \notin L_{r}$ for $S_{r}-T_{r}<t<0$, and $T_{r}-S_{r}>2 S_{r}=P_{r}$, a contradiction. Proposition 2.1 is proved. 


\section{Proof of Theorem 1.1}

Let $f$ be a transcendental meromorphic function in the plane with finitely many poles. Write $f=B / C$, where $B$ is a transcendental entire function and $C$ is a polynomial, having no zeros in common with $B$. The Wiman-Valiron theory [10] may now be applied to $B$ as follows. Starting from the Maclaurin series $B(z)=\sum_{k=0}^{\infty} b_{k} z^{k}$ of $B$, the central index $N(r)=\nu(r, B)$ is defined for $r \geq 0$ to be the largest integer $n$ such that $\left|b_{n}\right| r^{n}=\max _{k}\left|b_{k}\right| r^{k}$, and $N(r)$ tends to infinity with $r$. For large $r>0$ choose $z_{r}$ with $\left|z_{r}\right|=r$ and $\left|B\left(z_{r}\right)\right|=M(r, B)=\max \{|B(z)|:|z|=r\}$. Then [10, Theorem 10] gives $F_{1} \subseteq[1, \infty)$ such that $[1, \infty) \backslash F_{1}$ has finite logarithmic measure and

$$
\frac{f(z)}{f\left(z_{r}\right)} \sim \frac{B(z)}{B\left(z_{r}\right)} \sim\left(\frac{z}{z_{r}}\right)^{N(r)} \quad \text { on } D\left(z_{r}, 8\right),
$$

as $r \rightarrow \infty$ in $F_{1}$, where $D\left(z_{r}, 8\right)$ is given by (4).

Now Lemma 2.1 and Proposition 2.1 give an arbitrarily large number of pairwise disjoint trajectories for (1), each tending to infinity or a pole of $f$ in finite increasing time. But each of the finitely many poles of $f$ has only finitely many trajectories tending to it in increasing time (see Section 2). This proves Theorem 1.1.

It seems conceivable that the conclusion of Theorem 1.1 would remain true for all meromorphic functions $f$ in the plane such that the inverse function $f^{-1}$ has a direct transcendental singularity over $\infty$ [1]. This is a weaker hypothesis than those of Theorems 1.1 and 1.2, and means that there exist $M>0$ and a component $U$ of the set $\{z \in \mathbb{C}:|f(z)|>M\}$ which contains no poles of $f$, but does contain a path tending to infinity on which $f(z)$ tends to infinity. In this case, Theorems 2.1 and 2.2 of [2] give $F_{1}$ and $N(r)$ such that (2) and (3) are satisfied, where $\left|z_{r}\right|=r, D\left(z_{r}, 8\right) \subseteq U$ and $\log r=o\left(\log ^{+}\left|f\left(z_{r}\right)\right|\right)$ as $r \rightarrow \infty$ in $F_{1}$, while $[1, \infty) \backslash F_{1}$ has finite logarithmic measure. Thus Proposition 2.1 may be applied, with $P_{r} \rightarrow 0$ as $r \rightarrow \infty$ in $F_{1}$, by (5), but in general it seems difficult to exclude the possibility that all the trajectories $\zeta_{Y_{j}}$ thereby obtained tend to poles of $f$. It is true, however, that if such a trajectory does tend to a pole then it must exit $U$ and subsequently enter another component $U_{r}$ of $\{z \in \mathbb{C}:|f(z)|>M\}$, giving rise to an interval $\left[t_{1}, t_{2}\right] \subseteq\left(0, \beta_{Y_{j}}\right) \subseteq\left(0, P_{r}\right)$ on which $\left|f\left(\zeta_{Y_{j}}(t)\right)\right| \leq M$, with $\zeta_{Y_{j}}\left(t_{1}\right) \in \partial U$ and $\zeta_{Y_{j}}\left(t_{2}\right) \in \partial U_{r}$. Hence the distance from $U$ to $U_{r}$ is at most $M\left(t_{2}-t_{1}\right) \leq M P_{r}$, which for large $r \in F_{1}$ is extremely small. Such a component $U_{r}$ cannot exist if, for example, $f(z)=g(z) \tan z$, where $g$ is a transcendental entire function which is bounded on the strip $\{z \in \mathbb{C}:|\operatorname{Im} z| \leq T\}$, for some $T>0$; in this case $f^{-1}$ has a direct transcendental singularity over $\infty$ and (1) has infinitely many trajectories tending to infinity in finite increasing time.

\section{Proof of Theorem 1.3}

Let $f, \Omega, M, U, F$ and $H$ be as in the hypotheses. It may be assumed that $M=1$ : if this is not the case then (1) and $\Omega$ may be re-scaled by writing $w=z / M$ and $\dot{w}=f(z) / M=g(w)$. Let $z=\phi(v)$ be the inverse function of $F$, mapping $H=\{v \in \mathbb{C}: \operatorname{Re} v>0\}$ univalently onto $U$, and on $H$ consider the flow

$$
\phi^{\prime}(v) \dot{v}=e^{v} .
$$

The essence of the proof lies in showing that, since $\phi^{\prime}(v)$ varies relatively slowly on $H$, there are trajectories for (13) in $H$ which tend to infinity in finite time, and these are mapped via $z=\phi(v)$ to trajectories of (1) which tend to the extended boundary of $\Omega$. 
For $v \in H$ the function

$$
h(u)=\frac{\phi(v+u \operatorname{Re} v)-\phi(v)}{\phi^{\prime}(v) \operatorname{Re} v}=u+\sum_{n=2}^{\infty} a_{n} u^{n}
$$

is univalent for $|u|<1$, so that Bieberbach's theorem gives $\left|h^{\prime \prime}(0)\right|=2\left|a_{2}\right| \leq 4$ and

$$
\left|\frac{\phi^{\prime \prime}(v)}{\phi^{\prime}(v)}\right| \leq \frac{4}{\operatorname{Re} v} \quad \text { and } \quad\left|\log \left(\frac{\phi^{\prime}(s)}{\phi^{\prime}(v)}\right)\right| \leq \frac{C_{0} R}{\operatorname{Re} v} \quad \text { for } \quad|s-v|<R<\frac{\operatorname{Re} v}{2},
$$

where $C_{0}$ is a positive absolute constant. Moreover, there exists $C_{1}>0$ with

$$
\int_{[v,+\infty)} e^{-t}\left|\phi^{\prime}(t)\right| d t \leq \frac{\left|\phi^{\prime}(v)\right|}{v^{4}} \int_{[v,+\infty)} t^{4} e^{-t} d t \leq C_{1} e^{-v}\left|\phi^{\prime}(v)\right|
$$

for $v \in[1,+\infty) \subseteq \mathbb{R}$. Therefore, for $w \in H$, Cauchy's theorem and (15) lead to

$$
\begin{aligned}
D & =\int_{[1,+\infty)} e^{-t} \phi^{\prime}(t) d t \in \mathbb{C} \\
\int_{1}^{w} e^{-t} \phi^{\prime}(t) d t & =D-\psi(w)=D-\int_{w}^{+\infty} e^{-t} \phi^{\prime}(t) d t .
\end{aligned}
$$

Here the integral from 1 to $w$ is along any piecewise smooth contour in $H$, while that from $w$ to $+\infty$ is eventually along an interval $\left[M_{w},+\infty\right)$ with $M_{w} \geq 1$, and $\psi(w)$ is analytic on $H$.

Let $N_{1}$ and $N_{2} / N_{1}$ be large and positive, and for $j=1,2$ let $H_{j}$ denote the convex domain

$$
H_{j}=\left\{x+i y: x>N_{j},-x^{1 / 2 j}<y<x^{1 / 2 j}\right\} \subseteq H .
$$

Let $w$ lie in $H_{1}$, and write

$$
x=\operatorname{Re} w, \quad s=x+\sqrt{x} .
$$

Then (14), (15) and (17) imply that

$$
\begin{aligned}
\phi^{\prime}(w) & \sim \phi^{\prime}(x) \sim \phi^{\prime}(s), \\
\left|\int_{[s,+\infty)} e^{-t} \phi^{\prime}(t) d t\right| & \leq C_{1} e^{-s}\left|\phi^{\prime}(s)\right|=o\left(\left|e^{-w} \phi^{\prime}(x)\right|\right) .
\end{aligned}
$$

Further, the integral over the line segment from $w$ to $s$ satisfies, by (14) and (17),

$$
\int_{w}^{s} e^{-t} \phi^{\prime}(t) d t=\phi^{\prime}(x) \int_{w}^{s} e^{-t}(1+o(1)) d t=\phi^{\prime}(x)\left(e^{-w}-e^{-s}+\eta(w)\right),
$$

in which parametrising with respect to $\rho=\operatorname{Re} t$ gives

$$
|\eta(w)|=\left|\int_{w}^{s} e^{-t} o(1) d t\right| \leq o(1) \int_{x}^{s} e^{-\rho} d \rho=o\left(e^{-x}\right)=o\left(\left|e^{-w}\right|\right) .
$$

Combining the last estimate with (14), (16), (17), (18) and (19) leads to

$$
\psi(w) \sim e^{-w} \phi^{\prime}(x), \quad \lambda(w)=-\log \psi(w)=w+O(\log |w|)
$$

as $w \rightarrow \infty$ in $H_{1}$. Since $N_{2} / N_{1}$ is large, (14), (20) and Cauchy's estimate for derivatives yield $\left|\lambda^{\prime}(w)-1\right|<1 / 2$ on $H_{2}$, which implies that $\lambda(w)$ is univalent on $H_{2}$. Let $N_{3}$ and $N_{4}$ be positive integers with $N_{3} / N_{2}$ and $N_{4} / N_{3}$ large. Then (20) shows that for $j=0, \ldots, N_{3}$ there exists a simple path $L_{j}$ tending to infinity in $H_{2}$ and mapped by $\lambda$ onto the path $\left\{j 2 \pi i+t: t \geq N_{4}\right\}$. Thus $\psi=e^{-\lambda}$ maps each $L_{j}$ injectively onto $(0, h]$, where $h=e^{-N_{4}}>0$; moreover $\psi(v) \rightarrow 0$ and $e^{v} \rightarrow \infty$ as $v \rightarrow \infty$ on $L_{j}$. 
Parametrise $L_{j} \subseteq H_{2} \subseteq H$ by $w=v(s)$, where $-\psi(v(s))=s$ for $-h \leq s<0$. Thus

$$
1=-\psi^{\prime}(v(s)) \frac{d v}{d s}=e^{-v(s)} \phi^{\prime}(v(s)) \frac{d v}{d s},
$$

using (16), and so there exist $N_{3}$ pairwise disjoint trajectories $L_{j}$ in $H$ of the flow (13), on which $v$ and $e^{v}$ tend to infinity as $s \rightarrow 0-$ and so in finite increasing time.

Thus the flow (13) has infinitely many disjoint trajectories $L$ in $H$, on each of which $v(t)$ and $e^{v(t)}$ tend to infinity in finite increasing time. Because $\phi$ is univalent, these trajectories have disjoint images under $\phi$ in $U$. For each such trajectory $L$, write

$$
z=\phi(v), \quad \dot{z}=\phi^{\prime}(v) \dot{v}=e^{v}=e^{F(z)}=f(z) .
$$

Thus $f(z(t))$ tends to infinity in finite increasing time along $\phi(L) \subseteq U$, and it remains only to show that $z(t)$ tends to the extended boundary of $\Omega$. Assume that this is not the case: then there exists a sequence $\left(v_{j}\right) \subseteq L$ such that $e^{v_{j}}$ tends to infinity but $\beta_{j}=\phi\left(v_{j}\right) \rightarrow \beta_{0} \in \Omega$ as $j \rightarrow \infty$. Because $f\left(\beta_{j}\right)=e^{v_{j}} \rightarrow \infty$, it must be the case that $\beta_{0}$ is a pole of $f$ in $\Omega$. But then there exist a large positive $M_{1}$ and a neighbourhood $U_{1}$ of $\beta_{0}$ such that the closure of $U_{1}$ lies in $\Omega$ and $f$ maps $U_{1} \backslash\left\{\beta_{0}\right\}$ finite-valently onto $\left\{w \in \mathbb{C}: M_{1}<|w|<+\infty\right\}$. For large $j$ the line $\operatorname{Re} v=\operatorname{Re} v_{j}$ is mapped by $z=\phi(v)$ onto a level curve $\Gamma \subseteq U$ on which $|f|$ is constant, and $\Gamma$ passes through $\beta_{j} \in U_{1}$ and so must lie wholly in $U_{1}$. On the other hand, by the univalence of $\phi$, the level curve $\Gamma$ contains infinitely many distinct points $\phi\left(v_{j}+k 2 \pi i\right), k \in \mathbb{Z}$, each satisfying $f\left(\phi\left(v_{j}+k 2 \pi i\right)\right)=e^{v_{j}}=f\left(\beta_{j}\right)$. This proves Theorem 1.3.

Acknowledgement. The author thanks the referee for a very careful reading of the manuscript and extremely helpful suggestions.

\section{REFERENCES}

[1] W. Bergweiler and A. Eremenko, On the singularities of the inverse to a meromorphic function of finite order, Rev. Mat. Iberoamericana 11 (1995), 355-373.

[2] W. Bergweiler, P.J. Rippon and G.M. Stallard, Dynamics of meromorphic functions with direct or logarithmic singularities, Proc. London Math. Soc. 97 (2008), 368-400.

[3] L. Brickman and E.S. Thomas, Conformal equivalence of analytic flows, J. Differential Equations 25 (1977), 310-324.

[4] K.A. Broughan, The structure of sectors of zeros of entire flows, Topology Proceedings 27 (2003), 379-394.

[5] A. Garijo, A. Gasull and X. Jarque, Local and global phase portrait of equation $\dot{z}=f(z)$, Discrete Contin. Dyn. Syst. 17 (2007), 309-329.

[6] G. Gundersen, Estimates for the logarithmic derivative of a meromorphic function, plus similar estimates, J. London Math. Soc. (2) 37 (1988), 88-104.

[7] O. Hájek, Notes on meromorphic dynamical systems I, Czechoslovak Math. J. 16 (91) (1966), 14-27.

[8] O. Hájek, Notes on meromorphic dynamical systems II, Czechoslovak Math. J. 16 (91) (1966), 28-35.

[9] O. Hájek, Notes on meromorphic dynamical systems III, Czechoslovak Math. J. 16 (91) (1966), 36-40.

[10] W.K. Hayman, The local growth of power series: a survey of the Wiman-Valiron method, Canad. Math. Bull. 17 (1974) 317-358.

[11] W.K. Hayman, Subharmonic functions Vol. 2, Academic Press, London, 1989.

[12] D.J. Needham and A.C. King, On meromorphic complex differential equations. Dynam. Stability Systems 9 (1994), 99-122.

[13] R. Nevanlinna, Eindeutige analytische Funktionen, 2. Auflage, Springer, Berlin, 1953.

School of Mathematical Sciences, University of Nottingham, NG7 2RD, UK

E-mail address: james.langley@nottingham.ac.uk 